\title{
Significance of Renal Perfusion Angiography and Biochemical Indicators in Early Diagnosis of Type 2 Diabetic Nephropathy
}

\author{
Huiping Liang ${ }^{1, \dagger}$, Tingting $\mathrm{Lu}^{2, \dagger}$, Huaying Liu ${ }^{1}, \mathrm{Lili}_{\mathrm{Tan}^{2}}{ }^{2} \mathrm{Li} \mathrm{Li}^{1}$, Xiaojun Tang ${ }^{2, *}$ \\ ${ }^{1}$ Department of Medicine, Guangxi Medical College, Nanning, PR China \\ ${ }^{2}$ Department of Medical Technology, Guangxi Medical College, Nanning, PR China \\ Email address: \\ tangxiaojun008@163.com (Xiaojun Tang) \\ $\dagger$ Huiping Liang and Tingting Lu are co-first authors. \\ ${ }^{*}$ Corresponding author
}

To cite this article:

Huiping Liang, Tingting Lu, Li Li, Xiaojun Tang. Significance of Renal Perfusion Angiography and Biochemical Indicators in Early Diagnosis of Type 2 Diabetic Nephropathy. Pathology and Laboratory Medicine. Vol. 3, No. 1, 2019, pp. 5-9. doi: 10.11648/j.plm.20190301.12

Received: November 5, 2018; Accepted: December 14, 2018; Published: January 21, 2019

\begin{abstract}
Background: Although there are many biochemical indicators to reflect changes in renal function, but these indicators can not reflect renal damage timely and accurately. Renal microcirculation changes can not be reflected also. This study amied at evaluate significance of renal perfusion angiography and the indexes of relevant biochemical in early diagnosis of type 2 diabetic nephropathy. Methods: 30 I-III T2DN patients (DN group) and 25 healthy volunteers (control group) were studied, and both of groups were performed renal perfusion $\mathrm{CT}$ angiography imaging examination. Biochemical indexes, which are fasting blood glucose (FBG), urinary albumin excretion rate (UAE), $24 \mathrm{~h}$ urine protein quantitation (UPQ), blood urea nitrogen (BUN), serum creatinine (Scr), Cystatin C(Cys C), random albumin to creatinine ratio(ACR), were measured in both of groups, then simplified MDRD (Modification of Diet in Renal Disease) and Cys C equation were used to calculate the Estimated Glomerular Filtration Rate(eGFR). double-renal perfusion contrast scanning in both of groups were also conducted to obtain data of double renal blood flow (BF), blood volume (BV), mean transit time (MTT), and permeability surface (PS) for statistical analysis. Results: The indexes of BF, MTT and FBG, UAE, $24 \mathrm{~h} \mathrm{UPQ}, \mathrm{ACR}$, Cys C, eGFR were found to have relative strong correlation and statistical significance $(\mathrm{P}<0.05)$. BF was shown the negative correlation with those indexes, however MTT had positive correlation with them. The area under ROC (receiver operating characteristic curve), were more than 0.9 for UAE, ACR, $\mathrm{NF}$, and MTT, and it indicate that these indexes have relatively positive effect on the early diagnosis of the DN. For $24 \mathrm{~h} \mathrm{UPQ}$, cysteine C, eGFR and BV value, the area Under the curve are between 0.7 and 0.9 , which indicates that diagnosis using those index for the disease were accuracy. Thus, the above indexes have better diagnostic efficiency compared to Scr, BUN and eGFR. Conclusion: The biochemical indexes such as UAE, ACR and renal perfusion indexes such as BF and MTT can be applied in the early predicative diagnosis and DN screening. Its diagnosis effect is better than other biochemical indexes.
\end{abstract}

Keywords: Type 2 Diabetes Mellitus, Diabetic Nephropathies, Renal Function, Tomography, Spiral Computed, Perfusion

\section{Introduction}

Diabetic nephropathy (DN) is one of the most common complication of type 2 diabetes mellitus (T2DM), it is estimated that approximately $20-40 \%$ of type 2 diabetes (T2D) patients will develop renal disease [1]. Early features include slight increase in urinary albumin excretion which may eventually develop into end-stage renal disease. For diagnosis and the "gold standard" staging of DN, urinary albumin excretion rate (UAE) and estimated glomerular filtration rate (eGFR) is currently used. The incidence rate of diabetic patients of eGFR $<60 \mathrm{ml} /\left(\min \cdot 1.73 \mathrm{~m}^{2}\right)$ in china is $19.1 \%$, and micro albuminuria incidence rate was $17.3 \%$ [2-3]. However, the prognostic value of MA as a biomarker of disease progression is questionable, as patients with MA may exhibit the preservation of renal function[1]. There are researchs 
showing that the common used UAE, blood urea nitrogen ( BUN), serum creatinine(Scr) and other indicators for the early diagnosis of diabetic nephropathy has limitations on indications of renal damage, and with the development of medicine, multi-index comprehensive evaluation is more effective to improve the accuracy of clinical diagnosis [4]. Therefore, the identification of reliable biomarkers for the early prediction of DN is necessary. In the study, we used multilayers spiral CT functional imaging technique to obtain the renal perfusion parameters and to study the correlation between the parameters and related biochemical index for evaluating the significance of early diagnosis in DN.

\section{Materials and Methods}

\subsection{General Data}

Study was conducted in the First Affiliated Hospital of Guangxi Medical University from 2011 September to 2017 March. 55 cases were performed for renal CT perfusion imaging, including 30 cases patients with T2DM and early DN and 25 cases healthy volunteers. The Diagnosis of T2DM and DN accord to china standard guidelines for the prevention and treatment of type 2 diabetes formulated in 2010, and the patients who had hypertension, heart disease, severe liver and kidney dysfunction, ketoacidosis, hyperosmolar coma, the iodine agent allergy and performed angiography within 10 days were excluded. In DN group, 14 cases were male, and 16 cases were female, and mean age was $(57.7 \pm 7.3)$ yrs. The control group contained 13 male cases, 12 female cases, and mean age was $(56.7 \pm 7)$ yrs. There are no statistical difference of gender $(t=0.039, P=0.843)$ and age $(t=0.552, P=0.583)$ between two groups. The research was performed following the approval of ethical committee of the First Affiliated Hospital of Guangxi Medical University and the consent agreements were signed by all the participants.

\subsection{Detection of Related Biochemical Indices}

The day before the renal CT angiography examination, fasting blood glucose (FBG), urinary albumin excretion rate, $24 \mathrm{~h}$ urinary protein quantitation (UPQ), blood urea nitrogen, serum creatinine, Cystatin $\mathrm{C}$ (Cys $\mathrm{C}$ ) and random urine albumin to creatinine ratio (ACR) were measured on both of DN group and control group then the Scr value were conversed based on the new-old unit coefficient 0.01131 and further used to estimate eGFR by using equation: eGFR-MDRD $=175 \times(\mathrm{Scr}(\mathrm{mg} / \mathrm{dl}))^{-1.234} \times \quad \times \quad \mathrm{age}^{-0.179}$ $\times($ female $\times 0.79)$; the $\mathrm{Cys} C$ value were used to estimate eGFR based on the equations eGFR-cys $=78.64 \times \mathrm{Cys}^{-0.964}[1,5,6]$.

\subsection{Renal CT Perfusion Imaging Methods}

Before the examination, the patients underwent nil per oral $(\mathrm{N}$. P. O) for 12 hours, abdominal breathing training, iodine allergy test and standards water scheme. During the examination, patients were in the supine position, and shallow abdominal breathing, bellyband were fixed, chest, thyroid and pelvis and reproductive organs area were covered by protective clothing.

Two groups chose cubital vein for injecting $50 \mathrm{ml}$ of contrast agent (Ultravist 300, iodine $300 \mathrm{mg} / \mathrm{ml}$ ) with the speed of $4.0 \mathrm{ml} / \mathrm{s}$. After 6 seconds of injecting, 64 slices spiral CT (GE Light Speed VCT ) was posited at the both kidney level for renal perfusion scanning.

The data was uploaded to AW4.3 work station and used to calculate renal cortex blood flow (BF), blood volume (BV), mean transit time (MTT) of contrast agent and vascular surface permeability (permeability surface area product, PS) with software (renal perfusion model CT perfusion 3), then the average value for volume of kidney perfusion on cortex level were recorded.

\subsection{Statistical Analysis}

Statistical analysis were performed with SPSS 13, data were expressed as mean $\pm \mathrm{SD}$, and the difference between the groups were analyzed by using " $t$ " test; correlation analysis among parameters were studied by Pearson correlation analysis; receiver operating characteristic curve (ROC curve) was used for diagnostic performance evaluation.

\section{Results}

The comparison results of analysis on biochemical indicators between normal and diabetic nephropathy group was obtained. The clinical biochemical indexes of FBG, urinary albumin excretion rate (UAE), $24 \mathrm{~h}$ urinary protein quantitation $(24 \mathrm{~h}$ UPQ), urinary albumin/ creatinine ratio (ACR), Cys C and Cys C equation to estimate eGFR (eGFR-Ccys) showed statistically significant differences $(P<0.05)$; Scr, BUN and improved simplified MDRD equations in estimating eGFR (eGFR-MDRD) have no significant difference $(P>0.05)$ (Table 1).

Table 1. Comparison on biochemical indexes between subjects from two groups.

\begin{tabular}{|c|c|c|c|c|c|c|}
\hline & \multirow{2}{*}{$n$} & FBG & UAE & 24h UPQ & Scr & BUN \\
\hline & & $(\mathrm{mmol} / \mathrm{L})$ & $(\mathrm{mg} / 24 \mathrm{~h})$ & $(\mathrm{mg} / 24 \mathrm{~h})$ & $(\mu \mathrm{mol} / \mathrm{L})$ & $(\mathrm{mmol} / \mathrm{L})$ \\
\hline Control group & 25 & $5.17 \pm 0.69$ & $12.92 \pm 3.50$ & $88.26 \pm 26.23$ & $92.20 \pm 18.44$ & $5.16 \pm 1.24$ \\
\hline DN group & 30 & $11.38 \pm 2.65 * *$ & $73.28 \pm 39.31 * *$ & $161.76 \pm 64.53 * *$ & $99.87 \pm 38.82$ & $5.81 \pm 1.18$ \\
\hline
\end{tabular}

Table 1. Continued.

\begin{tabular}{|c|c|c|c|c|c|}
\hline & \multirow{2}{*}{$n$} & ACR & Cys C & eGFR-MDRD & eGFR-cys \\
\hline & & $(\mathrm{mg} / \mathrm{mmol})$ & $(\mathrm{mg} / \mathrm{L})$ & {$\left[\mathrm{ml} \cdot \min ^{-1}\left(173 \mathrm{~m}^{2}\right)^{-1}\right]$} & \\
\hline Control group & 25 & $1.39 \pm 0.40$ & $0.99 \pm 0.23$ & $72.26 \pm 25.57$ & $84.38 \pm 23.02$ \\
\hline DN group & 30 & $5.50 \pm 2.89 * *$ & $1.34 \pm 0.49 * *$ & $64.28 \pm 27.26$ & $67.89 \pm 26.24 *$ \\
\hline
\end{tabular}

VS Control group: $* P<0.05, * * P<0.01$. 
The analysis result of renal perfusion index between control group and diabetic nephropathy groups, where the perfusion of the contrast agent BV, BF, along with the MTT and PS showed statistically significant differences $(P<0.05)$ ( Table 2).

Table 2. Comparison on renal perfusion indexes between subjects from two groups.

\begin{tabular}{|c|c|c|c|c|c|}
\hline & & BV & MF & MTT & PS \\
\hline & $n$ & $(\mathrm{ml} / \mathbf{1 0 0 g})$ & $\left(\mathrm{ml} \cdot \mathrm{min}^{-1} \cdot 100 \mathrm{~g}^{-1}\right)$ & (S) & $\left(\mathrm{ml} \cdot \mathrm{min}^{-1} \cdot 100 \mathrm{~g}^{-1}\right)$ \\
\hline Control group & 25 & $39.55 \pm 9.50$ & $413.44 \pm 19.42$ & $5.75 \pm 0.86$ & $54.35 \pm 6.14$ \\
\hline DN & 30 & $34.64 \pm 8.58^{*}$ & $274.28 \pm 68.66^{* *}$ & $11.41 \pm 3.90 * *$ & $44.89 \pm 16.85 * *$ \\
\hline
\end{tabular}

VS Control group: $* P<0.05, * * P<0.01$.

The study result of analysis of correlation for the Renal perfusion and DN clinical biochemistry index, shows both renal perfusion contrast indexes BF, MTT along with the biochemical indices have a strong correlation with FBG, UAE, 24 h UPQ, ACR, Cys C, eGFR-cys and statistically significant $(P<0.05)$. There is a correlation with BUN, which is statistically significant $(P<0.05)$, but the correlation is weak with no statistical difference $(P>0.05)$ in Scr, eGFR-MDRD . There is a correlation with PS value and FBG, UAE, $24 \mathrm{~h}$
UPQ, ACR with statistical significance $(P<0.05)$, whereas there is weak correlation of $\mathrm{BV}$ value with $\mathrm{Cys} C$, with statistical significance $(P<0.05)$. These results suggest that $\mathrm{BF}$, MTT value and biochemical index for the diagnosis of diabetic nephropathy have better correlation. There is a negative correlation between the biochemical index and BF value, however there is positive correlation between the biochemical index and MTT value (Table 3).

Table 3. Analysis result of correlation between each renal perfusion index and DN clinical biochemical index.

\begin{tabular}{|c|c|c|c|c|c|c|c|c|}
\hline \multirow{2}{*}{$\begin{array}{l}\text { Pearson } \\
\text { test }(n=55)\end{array}$} & \multicolumn{2}{|l|}{ BV } & \multicolumn{2}{|l|}{ BF } & \multicolumn{2}{|l|}{ MTT } & \multicolumn{2}{|l|}{ PS } \\
\hline & $\begin{array}{l}\text { Correlation } \\
\text { coefficient }\end{array}$ & $p$-value & $\begin{array}{l}\text { Correlation } \\
\text { coefficient }\end{array}$ & $p$-value & $\begin{array}{l}\text { Correlation } \\
\text { coefficient }\end{array}$ & $p$-value & $\begin{array}{l}\text { Correlation } \\
\text { coefficient }\end{array}$ & $p$-value \\
\hline $\mathrm{FBG}$ & -0.255 & 0.061 & -0.723 & $<0.001$ & 0.633 & $<0.001$ & -0.410 & 0.002 \\
\hline 24h UPQ & -0.230 & 0.091 & -0.543 & $<0.001$ & 0.486 & $<0.001$ & -0.369 & 0.006 \\
\hline Scr & 0.071 & 0.604 & 0.030 & 0.826 & 0.010 & 0.942 & 0.204 & 0.136 \\
\hline BUN & -0.078 & 0.573 & -0.276 & 0.041 & 0.047 & 0.005 & 0.005 & 0.973 \\
\hline Cys C & -0.274 & 0.043 & -0.546 & $<0.001$ & 0.511 & $<0.001$ & -0.134 & 0.329 \\
\hline eGFR-MDRD & 0.024 & 0.863 & 0.047 & 0.735 & 0.090 & 0.515 & 0.037 & 0.122 \\
\hline eGFR-cys & 0.094 & 0.496 & 0.374 & 0.005 & -0.414 & 0.002 & 0.787 & 0.375 \\
\hline
\end{tabular}

The analysis result of diagnostic performance evaluation of ROC curves for each of the diagnostic index: clinical and biochemical indexes of FBG, UAE, $24 \mathrm{~h}$ urinary protein $(24 \mathrm{~h}$ UPQ), urinary albumin / creatinine ratio(ACR), Cys C and Cys $\mathrm{C}$ eGFR (eGFR-cys) estimation equation, renal perfusion index perfusion BV, BF, MTT of blood flow in the area under ROC curve values more than 0.5 have statistical significance $(\mathrm{P}<0.05)$ compared to control group, and all indexes can be used as a marker for early diagnosis of DN. The Biochemical index of Scr, BUN and improved simplified MDRD, equations in estimating eGFR (eGFR-MDRD) and renal vascular perfusion index surface permeability surface area product (PS) covers under the area of the ROC curve values more than 0.5 , but the difference was not statistically significant $(P>0.05)$, indicating that these indicators have difference in efficiency in early diagnosis of DN. the Area of FBG, UAE, ACR, BF, MTT Under the ROC curve were more than 0.9, showing these indicators are better for diagnosis of DN. $24 \mathrm{~h} \mathrm{UPQ}$, Cys C, eGFR-cys and BV value under the curve were between 0.7 0.9 , indicating a certain degree of accuracy in the diagnosis of DN. The above indicators have better accuracy for DN diagnosis than Scr, BUN and eGFR-MDRD (Table 4).

Table 4. Analysis result of diagnosis performance evaluation ROC curve of each diagnosis index.

\begin{tabular}{llllll}
\hline \multirow{2}{*}{ Index } & \multirow{2}{*}{ Area } & Standard Error & $\mathbf{9 5 \% C I}$ & & \\
\cline { 4 - 5 } & & & Lower Bound & Upper Bound & p-value \\
\hline FBG & 1.000 & 0.000 & 0.935 & 1.000 & $<0.001$ \\
UAE & 1.000 & 0.000 & 0.935 & 1.000 & $<0.001$ \\
24h UPQ & 0.848 & 0.050 & 0.726 & 0.931 & 0.001 \\
Scr & 0.528 & 0.081 & 0.389 & 0.664 & 0.728 \\
BUN & 0.645 & 0.075 & 0.505 & 0.770 & $<054$ \\
ACR & 1.000 & 0.000 & 0.935 & 1.000 & 0.001 \\
Cys C & 0.713 & 0.072 & 0.576 & 0.827 & 0.003 \\
eGFR-MDRD & 0.578 & 0.078 & 0.437 & 0.710 & 0.318 \\
eGFR-cys & 0.713 & 0.072 & 0.576 & 0.827 & 0.003 \\
BV & 0.715 & 0.076 & 0.577 & 0.748 & 0.005 \\
PS & 0.620 & 0.079 & 0.479 & 1.000 & $<1275$ \\
BF & 0.997 & 0.003 & 0.930 & 1.000 & $<0.001$ \\
MTT & 0.996 & 0.005 & 0.927 & & \\
\hline
\end{tabular}




\section{Discussion}

With the prolong survival duration of diabetic patients, T2DM caused increasingly complications, $20-40 \%$ diabetes patients occurs DN that is the main cause for renal failure. When $\mathrm{DN}$ is at the early stage, it usually has a good prognosis, once proteinuria occurs, the disease become irreversible and will finally develop into chronic renal failure [7].

UAE, SCr and BUN are commonly used for early diagnosis of DKD, but they are difficult to judge in diabetic patients with early renal injury [4]. Excretion of urine, albumin and creatinine in the urine can be fluctuated by some factors, therefore, in the same individual, ACR can remain relatively constant. In this study, random urine UAE performance evaluation and ACR are in ROC curve analysis area. The area under the curve is equal to or more than 0.9 , indicating that these parameters of DN has a very good diagnostic efficacy and is better than $24 \mathrm{~h} \mathrm{UPQ}$, Scr, and BUN based on Chinese improvement simplified MDRD equations which estimates the glomerular filtration rate (eGFR-MDRD). In the traditional 24 hour urinary albumin excretion rate method, the sample collection process is long, and the results affected by patient's susceptible to compliance. The ACR measurement using randomly collected urine is quicker, more simple and reliable, and can be used as an important index for early diagnosis of diabetic kidney disease. The 2013 guidelines for T2DM prevention and treatment released by the National Institute for Health and Care Excellence (NICE) recommends to use ACR for early diabetic renal damage detection and staging [8].

Researchs have shown that the damage of renal function, urine protein and ACR changes are not always consistent, about $20 \% \sim 30 \%$ of T2DM patients have renal function damage before showing any change in urinary protein. And Proteinuria are affected by many factors, such as sport, bleeding and infection and so on, therefore, only using UAE and ACR index to monitor the DN is not enough. Current widely used indexes for renal function evaluation include Scr, BUN and glomerular filtration rate based on Scr. However, the renal function is affected not only by plasma Scr, BUN concentration and also by body metabolism, blood volume status, diet, drugs and other factors. Studies have shown that the difference of Scr serum level is no significant between the normal renal function stage and albumin phase, and becomes significant until DN IV stage, which limits the Practicability of Scr as a clinical marker for evaluating GFR [9]. Our results showed that Scr, BUN and Scr based eGFR-MDRD did not have diagnosis significance. Cys $\mathrm{C}$ in the blood serum can be freely filtered through the glomerulus, renal tubule and decomposes completely in the brocade of renal tubule, and its blood concentration entirely depends on the GFR and is not easily to be influenced by other factors. Many study have shown that CysC is better than Scr and BUN as an ideal endogenous index, which meet the requirement for evaluating GFR and can be used as a accuracy and reliable clinical index for the early diagnosis of diabetic renal damage [10]. Zhang, et al confirmed that Cys $\mathrm{C}$ is a better index than Scr for evaluating GFR in DN patients on high perfusion stage and final stage of the renal insufficiency stage [11]. NICE also pointed out that Cys $\mathrm{C}$ is more effective to assess GFR than Scr in GFR $>45\left(\mathrm{ml} \cdot \mathrm{min}^{-1}\left(173 \mathrm{~m}^{2}\right)^{-1}\right)$ and without proteinuria patients, and is an alternative endogenous filtration markers [8]. Our study included the normal volunteers and early DN (DN I-III period) patients, their serum Cys $\mathrm{C}$ were detected and used in the equation for calculating eGFR (eGFR-Ccys) to evaluate the prevalence of DN patients. The area of under ROC curves of Cys C and eGFR-Ccys are between 0.7 0.9, indicatingthat its accuracy is better than Scr, BUN and eGFR-MDRD in the diagnosis of DN.

Although there are many clinical biochemical indexes using to reflect the renal function changes, these indicators have the limitation with low sensitivity and specificity, especially during compensatory period, these indicators may not accurately reflect the situation of kidney damage, furthermore, those blood or urine tests only reflect renal situation indirectly and cannot directly reflect changes inside the renal parenchymal microcirculation. Renal biopsy can reflect directly in case of early pathological renal lesion, however the invasiveness and poor patient compliance makes it difficult to be used in the clinic. To early diagnose DN in I $\sim$ III early stage, there are still needs to find out some representative and predictive method.

Research showed that the renal cortex and medulla blood flow perfusion are damaged in hypertensive and diabetic nephropathy renal altered function, and the amount of blood perfusion of renal cortex is a direct index to judge the renal blood flow [12]. CT perfusion imaging was a proposed method in the early 90th century, and it can evaluate accurately renal function for clinical reference with clear anatomical image. The current used low osmolar non-ionic contrast agent for renal perfusion CT has very less impact on blood flow when breathing. Renal perfusion contrast can also be a measuring method for evaluating functional state of renal perfusion through $\mathrm{BF}, \mathrm{BV}$ and the changing time of contrast agents color (MTT) [13]. In our study, 25 healthy volunteers and 30 patients have been performed renal perfusion imaging based on the diagnostic criteria for early DN. The result show that BF and MTT of Renal perfusion angiography have a strong correlation with the accepted biochemical marker for DN including UAE, 24 hUPQ, ACR, CysC and eGFR-cys. It also has been shown the weak correlation with BUN and no correlation with Scr, eGFR-MDRD for early diagnosis of DN, and the results are consistant with the past finding. ${ }^{12}$ And indicated the decreased BF and prolong MTT in the CT perfusion imaging of early $\mathrm{DN}$, and reflected the increase of renal artery resistance, the changes of renal artery morphology and renal cortex perfusion, and the evaluation of glomerular filtration rate.

Nowadays, CT perfusion imaging is increasingly used for diagnosis of $\mathrm{DN}$ and other kidney disease. Research have shown that the risk of contrast-inducednephropathy (CIN) from contrast-enhanced MDCT in patients with renal 
insufficiency appeares to be low, and there is no significant difference in the incidence of CIN in comparison with patients who did not receive contrast medium (CM) [14].

Renal perfusion angiography can be used to evaluate renal function through providing excellent anatomical and morphological picture, thus, it need to be further investigated for clinical practice.

\section{Conclusions}

The biochemical indexes such as UAE and ACR and renal perfusion indexes such as, BF and MTT can be applied in the early predicative diagnosis and $\mathrm{DN}$ screening. Its diagnosis effect is better than other biochemical indexes.

\section{Disclosure Statement}

The authors declare that there are no conflicts of interest.

\section{Acknowledgements}

This work was supported by a grant from the basic ability promotion project for young and middle-aged teachers in Universities in Guangxi (No. 2018KY1146).

\section{References}

[1] Liao WL, Chang CT, Chen CC, Lee WJ, Lin SY, Liao HY, Wu CM, Chang YW, Chen CJ: Urinary Proteomics for the Early Diagnosis of Diabetic Nephropathy in Taiwanese Patients. Journal of Clinical Medicine 2018;7(12):483.

[2] Association AD: Standards of medical care in diabetes-2012. Diabetes Care 2012;35:S11-S63.

[3] Z S, P Z, C Z: Prevalence of chronic kidney disease in china. Lancet 2012;379:815-822.

[4] Philips JC, Marchand M, Scheen AJ: Pulse pressure and cardiovascular autonomic neuropathy according to duration of type 1 diabetes. Diabetes/metabolism Research \& Reviews 2009;25:442-451.
[5] National eGFR project collaboration group of China: Modification and evaluation of mdrd estimating equation for chinese patients with chronic kidney disease. Chinese Journal of Nephrology 2006;22:589-595.

[6] Feng J, Qiu L, Zhang L, Li X, Yang Y, Zeng P, Guo X, Qin Y, Liu H, Han X: Multicenter study of creatinine- and/or cystatin c-based equations for estimation of glomerular filtration rates in chinese patients with chronic kidney disease. Plos One 2013;8:e57240.

[7] Bembde AS: A study of plasma fibrinogen level in type-2 diabetes mellitus and its relation to glycemic control. Indian Journal of Hematology and Blood Transfusion 2012; 28: 105-108.

[8] Carville S, Wonderling D, Stevens P: Early identification and management of chronic kidney disease in adults: Summary of updated nice guidance. Bmj 2014;349:g4507.

[9] Macisaac RJ, Premaratne E, Jerums G: Estimating glomerular filtration rate in diabetes using serum cystatin c. Clinical Biochemist Reviews 2011;32:61-67.

[10] Assal HS, Tawfeek S, Rasheed EA, Ellebedy D, Thabet EH: Serum cystatin c and tubular urinary enzymes as biomarkers of renal dysfunction in type 2 diabetes mellitus. Clinical Medicine Insights Endocrinology \& Diabetes 2013;6:7.

[11] Zhang PP, Zhan JF, Xie HL, Li LS, Liu ZH: Evaluation of glomerular filtration rate using cystatin $\mathrm{c}$ in diabetic patients analysed by multiple factors including tubular function. Journal of International Medical Research 2010;38:473-483.

[12] Haynes R, Judge PK, Staplin N, Herrington WG, Storey BC, Bethel A, Bowman L, Brunskill N, Cockwell P, Hill M, Kalra PA, McMurray JJV, Taal M, Wheeler DC, Landray MJ, Baigent C: Effects of Sacubitril/Valsartan Versus Irbesartan in Patients With Chronic Kidney Disease. Circulation 2018; 138 (15): 1505-1514.

[13] Dawson P: Functional imaging in ct. European Journal of Radiology 2006;60:331-340.

[14] Murakami R, Hayashi H, Sugizaki K, Yoshida T, Okazaki E, Kumita S, Owan C: Contrast-induced nephropathy in patients with renal insufficiency undergoing contrast-enhanced mdct. European Radiology 2012;22:2147-2152. 